\title{
DNA methylation as a predictor of fetal alcohol spectrum disorder
}

\author{
Alexandre A. Lussier ${ }^{1,2}$, Alexander M. Morin ${ }^{1}$, Julia L. Maclsaac ${ }^{1}$, Jenny Salmon, ${ }^{3,}$, Joanne Weinberg², \\ James N. Reynolds ${ }^{5}$, Paul Pavlidis ${ }^{6,8}$, Albert E. Chudley $^{3,4}$ and Michael S. Kobor ${ }^{1,7^{*}}$
}

\begin{abstract}
Background: Fetal alcohol spectrum disorder (FASD) is a developmental disorder that manifests through a range of cognitive, adaptive, physiological, and neurobiological deficits resulting from prenatal alcohol exposure. Although the North American prevalence is currently estimated at 2-5\%, FASD has proven difficult to identify in the absence of the overt physical features characteristic of fetal alcohol syndrome. As interventions may have the greatest impact at an early age, accurate biomarkers are needed to identify children at risk for FASD. Building on our previous work identifying distinct DNA methylation patterns in children and adolescents with FASD, we have attempted to validate these associations in a different clinical cohort and to use our DNA methylation signature to develop a possible epigenetic predictor of FASD.
\end{abstract}

Methods: Genome-wide DNA methylation patterns were analyzed using the Illumina HumanMethylation450 array in the buccal epithelial cells of a cohort of 48 individuals aged 3.5-18 (24 FASD cases, 24 controls). The DNA methylation predictor of FASD was built using a stochastic gradient boosting model on our previously published dataset FASD cases and controls (GSE80261). The predictor was tested on the current dataset and an independent dataset of 48 autism spectrum disorder cases and 48 controls (GSE50759).

Results: We validated findings from our previous study that identified a DNA methylation signature of FASD, replicating the altered DNA methylation levels of 161/648 CpGs in this independent cohort, which may represent a robust signature of FASD in the epigenome. We also generated a predictive model of FASD using machine learning in a subset of our previously published cohort of 179 samples (83 FASD cases, 96 controls), which was tested in this novel cohort of 48 samples and resulted in a moderately accurate predictor of FASD status. Upon testing the algorithm in an independent cohort of individuals with autism spectrum disorder, we did not detect any bias towards autism, sex, age, or ethnicity.

Conclusion: These findings further support the association of FASD with distinct DNA methylation patterns, while providing a possible entry point towards the development of epigenetic biomarkers of FASD.

Keywords: Fetal alcohol spectrum disorder, Epigenetics, DNA methylation, Biomarkers, Neurodevelopmental disorders

\footnotetext{
* Correspondence: msk@cmmt.ubc.ca

'Department of Medical Genetics, Centre for Molecular Medicine and

Therapeutics, British Columbia Children's Hospital Research Institute,

University of British Columbia, Vancouver, British Columbia, Canada

${ }^{7}$ Human Early Learning Partnership, University of British Columbia, Vancouver,

British Columbia, Canada

Full list of author information is available at the end of the article
}

(c) The Author(s). 2018 Open Access This article is distributed under the terms of the Creative Commons Attribution 4.0 International License (http://creativecommons.org/licenses/by/4.0/), which permits unrestricted use, distribution, and reproduction in any medium, provided you give appropriate credit to the original author(s) and the source, provide a link to the Creative Commons license, and indicate if changes were made. The Creative Commons Public Domain Dedication waiver (http://creativecommons.org/publicdomain/zero/1.0/) applies to the data made available in this article, unless otherwise stated. 


\section{Background}

Fetal alcohol spectrum disorder (FASD) is a leading preventable cause of developmental disability, with a North American prevalence currently estimated at 2-5\% [1-3]. FASD presents through a wide spectrum of phenotypes, ranging from growth deficits and physical abnormalities to cognitive and behavioral deficits, as well as motor and sensory impairments, immune dysfunction, and increased vulnerability to mental health problems in adulthood [4-6]. On the most severe end of the spectrum lies fetal alcohol syndrome (FAS), which is characterized by growth retardation, a distinct set of facial dysmorphisms, and central nervous system abnormalities $[7,8]$. By contrast, Alcohol-Related Neurodevelopmental Disorder (ARND) describes the less visible and largest group within the spectrum, where individuals with confirmed alcohol exposure during pregnancy show primarily behavioral, adaptive, and/or cognitive abnormalities without obvious facial dysmorphisms [9]. Of note, individuals across the spectrum show cognitive and behavioral deficits, which can be as serious in those without any physical features as in those with full FAS [10].

Although children with FAS are often diagnosed in infancy or in early life, FASD in general has proven difficult to identify, particularly in the absence of the overt facial features characteristic of FAS. As such, many individuals with FASD are not identified until they reach school age, where they begin to struggle with increased social pressure and cognitive challenges [11]. However, early cognitive and behavioral interventions may potentially attenuate some of the deficits associated with FASD and improve the long-term outcomes of these individuals [12]. As early diagnosis is a strong predictor of positive outcome, early screening tools are necessary to help identify at-risk children at a young age and potentially buffer some of the deficits associated with prenatal alcohol exposure (PAE) [13, 14].

While self-report methods are most commonly used for assessing PAE and a child's risk for FASD, these are not always accurate and can underestimate alcohol consumption during pregnancy [15-17]. Over the past decades, various biomarkers of alcohol exposure have been developed to complement self-report measures, focusing primarily on the direct or indirect products of ethanol metabolism, which can be measured in biological specimens from both the mother and infant [18]. Although these biomarkers are very sensitive to alcohol exposure, they present a number of limitations when attempting to determine whether prenatal alcohol exposure has occurred or to gain insight into the biological underpinnings of alcohol-induced deficits and the developmental profiles associated with FASD. For example, many of these biomarkers have short windows of detection (e.g., urine, blood, plasma) or are limited by specimen availability (e.g., placenta, meconium), making them useful for identification of alcohol exposure around the time of parturition, but not in infants and children over the course of development [19]. As such, objective and persistent measures are needed to aid in the screening and diagnosis of children at risk for FASD.

Epigenetic marks are now emerging as potential biomarkers or signatures of early-life exposures. Broadly defined, epigenetics refers to modifications of DNA and its regulatory components, including chromatin and non-coding RNA, that potentially modulate gene transcription without changing underlying DNA sequences [20-22]. In addition to their role in the regulation of cellular processes, these may also bridge environmental factors and genetic regulation to capture a lasting signature of early life exposures. In particular, DNA methylation is emerging as a candidate biomarker for environmental exposures and disease. Typically found on the cytosine residues of cytosine-guanine dinucleotides $(\mathrm{CpG})$, this epigenetic mark is both stable over time and dynamic in response to environmental factors [23]. Several pre- and postnatal environmental influences have been associated with altered DNA methylation patterns, hinting at possible malleability by early-life environments and suggesting a potential utility as biomarkers [24, 25]. For example, prenatal exposure to cigarette smoke is associated with lasting alterations to DNA methylation patterns, which are now being used as biomarkers of cigarette smoke exposure in infants [26].

While in its infancy in relation to FASD, epigenetic biomarkers show promise for early screening of at-risk individuals, as the DNA methylome retains a lasting signature of prenatal alcohol exposure in both the central nervous system and peripheral tissues (reviewed in [27]). Numerous studies performed in animal and cell culture models have identified both short-term and persistent alterations to DNA methylation patterns following PAE. Although some of these models reflect supraphysiological levels of alcohol exposure or display modest effect sizes in response to PAE, the findings from these pre-clinical models suggest the possibility that PAE may directly influence epigenetic patterns and that these may play a role in PAE-induced deficits [27-33]. By contrast, fewer studies have investigated DNA methylation patterns in individuals with FASD. More targeted methods identified differences in DNA methylation levels in the promoter region of Drd4 in a large Australian cohort of children exposed to alcohol during breastfeeding [34]. Others have employed discoverydriven approaches, assessing genome-wide DNA methylation patterns in case-control studies of FASD. The first of these came from a small cohort of children, where slight differences in DNA methylation patterns within the protocadherin $(\mathrm{PCDH})$ gene clusters reported with a 
rather modest significance threshold [35]. Recently, we analyzed DNA methylation profiles in a large cohort of children with FASD recruited by NeuroDevNet (NDN), a Canadian Networks of Centres of Excellence, where we identified a signature of 658 differentially methylated CpGs [36]. Although few results have been validated across different cohorts, these findings set the stage for broader applications of DNA methylation in the context of FASD, creating a framework upon which to build future epigenomic studies of FASD.

To validate the findings from our previous DNA methylation study, we assessed the genome-wide DNA methylation profiles of buccal epithelial cells (BEC) from an independent cohort of 24 individuals with FASD, aged $3.5-18$, and 24 typically developing controls, aged 5-17. Given that our initial study provided a framework for genome-wide assessment of DNA methylation patterns in individuals with FASD, we used the findings from the NDN study as a foundation for the identification of replicable epigenetic differences associated with FASD. Notably, nearly $25 \%$ of statistically significant associations from the NDN cohort were validated in this new cohort at a false-discovery rate $(F D R)<0.05$ [37]. In addition to the validation analyses, we also assessed whether DNA methylation profiles could be used to identify individuals with FASD, generating a classification algorithm that uses DNA methylation levels to accurately predict FASD status. Taken together, these results suggested that there were replicable differences in DNA methylation patterns between individuals with FASD and controls, which could potentially contribute to the development of a screening tool for at-risk children.

\section{Methods}

\section{The Kids Brain Health Network cohort of children with} FASD

The present cohort was collected as a replication study by Kids Brain Health Network (KBHN), formerly NeuroDevNet, and is hereby referred to as the KBHN cohort [38]. Ethics for this study were reviewed and approved by the "Children's and Women's Research Ethics Board - Clinical” (H10-01149). All experimental procedures were reviewed and approved by the University of Manitoba and the University of British Columbia. Written informed consent was obtained from a parent or legal guardian, and assent was obtained from each child before study participation. The clinics used previously described guidelines for the diagnosis of FASD [39]. Children with FASD and typically developing controls were recruited from the Manitoba FASD diagnostic clinic in Winnipeg, Manitoba, Canada. Briefly, buccal epithelial cell (BEC) samples were collected for DNA methylation analysis from 25 FASD and 26 age- and sex-matched control children aged between 3.5 and 18 , prior to pre-processing (Table 1). BECs were collected using the Isohelix buccal swabs and Dri-Capsule (Cell Projects Ltd., Kent, UK). To collect buccal cells, the swab was inserted into the participants' mouth and rubbed firmly against the inside of the left cheek for $1 \mathrm{~min}$. The swab was then placed into a sterile tube with a Dri-Capsule and the tube sealed. An identical procedure was followed for the right cheek. Participants did not have any dental work performed $48 \mathrm{~h}$ prior to collection, and no food was consumed less than $60 \mathrm{~min}$ prior to collection to avoid contamination.

\section{DNA methylation $450 \mathrm{~K}$ assay}

DNA was extracted from BECs using the Isohelix DNA isolation kit (Cell Projects, Kent, UK). Seven hundred fifty nanograms of genomic DNA was subjected to bisulfite conversion using the Zymo EZ DNA Methylation Kit (Zymo Research, Irvine, California), which converts DNA methylation information into sequence base differences by deaminating unmethylated cytosines to uracil while leaving methylated cytosines unchanged. One hundred sixty nanograms of converted DNA was applied to the HumanMethylation450 BeadChip array from Illumina (450K array), which enables the simultaneous

Table 1 Characteristics of the NeuroDevNet II FASD cohort

\begin{tabular}{lll}
\hline & FASD cases & Controls \\
\hline$N$ & 24 & 24 \\
ARND & 18 & \\
Partial FAS & 6 & \\
FAS & 1 & \\
FASD & 1 & \\
Age (years) & & $5-17$ \\
Range & $3.5-18$ & 11.6 \\
Mean & 9.1 & \\
Sex & & 13 \\
Female & 9 & 11 \\
Male & 15 & 22 \\
Self-declared ethnicity & & 1 \\
Caucasian & $4(2)^{a}$ & 1 \\
First Nations & $17(20)^{a}$ & 0 \\
Asian & $1(0)^{a}$ & 0 \\
Not reported & 2 & 24 \\
Caregiver status & 6 & 0 \\
Biological parents & 7 & \\
Biological grandparents & 3 & \\
Adopted/legal guardian & 6 & \\
Foster care & & \\
\hline
\end{tabular}

Including mixed lineage First Nations 
quantitative measurement of 485,512 CpG sites across the human genome, following the manufacturer's instructions. Chips were scanned on an Illumina HiScan, with the 51 samples run in two batches and each containing a similar number of FASD and control samples, randomly distributed across the chips. Two pairs of technical replicates were also included and showed a Pearson correlation coefficient $r>0.994$ in both cases, highlighting the technology's reproducibility on our inhouse platform. Inter-sample correlations ranged from $0.926-0.99$.

\section{DNA methylation data quality control and normalization}

The raw DNA methylation data were subjected to a rigorous set of quality controls, first of the samples, and then of the probes. Of the 51 initial samples, 3 were removed from the final dataset based on poor quality data, which was identified through skewed internal controls and/or $>=5 \%$ of probes with a detection $p$ value $>0.05$ ( 2 controls and 1 FASD). Next, probes were removed from the dataset according to the following criteria: (1) probes on X and Y chromosomes $(n=11,648),(2)$ SNP probes $(n=65)$, (3) probes with bead count $<3$ in $10 \%$ of samples $(n=726),(4)$ probes with $10 \%$ of samples with a detection $p$ value $>0.01(n=11,864)$, and (5) probes with a polymorphic $\mathrm{CpG}$ and non-specific probes $(N=19,337$ SNP-CpG and 10,484 non-specific probes) [40]. A final filtering step was performed to set the methylation values to NA for any remaining probe-sample pair where bead count $<3$ or detection $p$ value $>0.01$. Data normalization was performed using the SWAN method on the final dataset, composed of 48 samples (24. FASD and 24 controls) and 431,544 probes [41]. Finally, batch effects (chip number and chip position) were removed using the ComBat function from the SVA package in $\mathrm{R}$ [42]. Statistical analyses were performed using on ComBat-corrected $M$ values, which represent the $\log 2$ ratio of methylated/unmethylated, where negative values indicate less than $50 \%$ methylation and positive values indicate more than 50\% methylation [43]. Percent methylation differences (beta-values) were used in graphical representations of the data and indicate the percentage of methylation calculated by methylated/ (methylated + unmethylated), ranging from 0 (fully unmethylated) to 1 (fully methylated).

\section{Differential methylation analysis and validation of NeuroDevNet (NDN) findings}

Cell type deconvolution was performed to assess the proportions of CD14, CD34, and buccal epithelial cells in each sample using DNA methylation levels at CpGs highly correlated with these cell types [44]. Surrogate variable analysis (SVA) was also performed on ComBatcorrected, normalized data using the SVA package in $\mathrm{R}$ to identify surrogate variables (SVs) representative of unwanted heterogeneity [42]. Using DNA methylation data from all 48 samples, SVA identified 6 SVs not associated with clinical status (FASD vs control). As these were partially associated with known covariates, such as cell type proportions and age, the SVs were included in the linear regression analysis to account for their effects. More specifically, linear modeling was performed on the 648 differentially methylated probes identified in the initial NDN study and found in the present dataset using the limma package in $\mathrm{R}$ and a model that included clinical status and all identified SVs as covariates [36, 45]. Significant differentially methylated probes between groups were identified at a false-discovery rate (FDR) < 0.05 following multiple test correction by the BenjaminiHochberg method and were required to show the same direction of change as the NDN cohort's findings [46]. Further evaluation of potential biological significance was performed using an arbitrary threshold of $>5 \%$ mean percent DNA methylation difference between FASD and controls.

\section{DNA methylation pyrosequencing assay}

The bisulfite pyrosequencing assay was designed with PyroMark Assay Design 2.0 (Qiagen; Additional file 1: Table S1). The region of interest was amplified by PCR using the HotstarTaq DNA polymerase kit (Qiagen) as follows: 15 min at $95{ }^{\circ} \mathrm{C}, 45$ cycles of $95{ }^{\circ} \mathrm{C}$ for 30 s, $58^{\circ}$ $\mathrm{C}$ for $30 \mathrm{~s}$, and $72{ }^{\circ} \mathrm{C}$ for $30 \mathrm{~s}$, and a 5 -min $72{ }^{\circ} \mathrm{C}$ final extension step. For pyrosequencing, single-stranded DNA was prepared from the PCR product with the Pyromark $^{\text {Tim }}$ Vacuum Prep Workstation (Qiagen) and the sequencing was performed using sequencing primers on a Pyromark ${ }^{\text {Tx }}$ Q96 MD pyrosequencer (Qiagen). The quantitative levels of methylation for each $\mathrm{CpG}$ dinucleotide were calculated with Pyro Q-CpG software (Qiagen).

\section{The NDN cohort of children with FASD}

DNA methylation data from the previous cohort of children with FASD were obtained from GEO (GSE80261) and normalized as described in our original publication [36]. This cohort was collected by NeuroDevNet, a Canadian Network of Centres for Excellence, and is hereby referred to as the NDN cohort [36]. Briefly, we selected the individuals with a confirmed diagnosis of FASD from this dataset, as well as age- and sex-matched typically developing controls, resulting in dataset composed of 83 children with FASD ( 55 ARND, 18 partial FAS, 10 FAS) and 96 typically developing controls. The mean age (in years) for individuals with FASD was 11.88 and 11.28 for controls, both ranging from 5 to 18 years old. The proportions of males and female differed slightly between groups, with 42 females and 41 males in 
the FASD cases and 57 females and 39 males in the control group. A skew in self-declared ethnicity was present between the groups, as the majority of controls identified as Caucasian, while the majority of children in the FASD group identified as First Nations. This skew was addressed in the initial epigenome-wide association study through the use of a more ethnically homogeneous subset of the cohort. DNA methylation data were obtained from BEC using the Illumina $450 \mathrm{~K}$ array and were normalized using the beta-mixture quantile normalization method.

\section{Cohort of individuals with autism spectrum disorder}

Processed DNA methylation data from a publically available dataset of individuals with autism spectrum disorder (ASD) were obtained from GEO (GSE50759). Briefly, this dataset was composed of 48 individuals with ASD and 48 typically developing controls. As per the authors' description of the GEO data, these were preprocessed using the $\mathrm{R}$ packages minfi and sva to obtain normalized $M$ values [47]. The samples consisted of 58 males and 38 females, consistent with the skew towards males in ASD. The mean age (8.84) and range (128 years old) differed from the NDN and KBHN studies, and the genetic ancestry of most individuals was Caucasian (European), though a proportion of the cohort was of Nigerian ancestry. DNA methylation data of these samples were obtained from BEC using the Illumina 450K array.

\section{DNA methylation as a predictor of FASD status}

A predictive model of FASD status was created using DNA methylation data and the caret package in $R$ [48]. First, a predictive model was created using stochastic gradient boosting on the NDN cohort (83 FASD cases, 96 controls) using the beta-values of the differentially methylated probes identified in the NDN study (648 probes) [36]. The parameters of the modeling were optimized for area under the receiver operating characteristic (ROC) curve by grid tuning for repeated cross-validation (number of trees 50-1500; 1, 5 , or 9 interaction depth; 0.1 shrinkage). The optimal model for predicting clinical FASD status using 648 probes was 550 trees, 1 of interaction depth, and 20 minimum observations per node. The KBHN cohort (24 FASD, 24 controls) was then used to verify the predictive sensitivity and specificity of the model. In parallel, $450 \mathrm{~K}$ data from a cohort of children with ASD were tested to verify the predictive specificity of the model for FASD. The predictor was tested using normalized data that was uncorrected for batch effects to better mimic the potential use of the predictive model by independent groups.

\section{Results}

\section{The KBHN cohort of children with FASD}

As noted, we analyzed genome-wide DNA methylation patterns from 24 children with FASD and 24 typically developing controls, matched for sex and age, ranging from 3.5 to 18 years of age (Table 1). We found that self-declared ethnicity, primary caregiver, and mean age were significantly different between the FASD and control participants (Student's $t$ test; $p<0.05$ ). We corrected for the potential effects of age on DNA methylation through the statistical methods outlined below. However, given the confound in self-declared ethnicity and caregiver status, we could not correct for these effects and relied on the previous correction of ethnic bias in the initial NDN study (see below) [36]. Furthermore, we could not account for the different life experiences of individuals with FASD, including potential exposure to adverse early life events at considerably higher levels than those in the general population. It is possible that these distinct experiences in themselves may potentially be associated with DNA methylation patterns.

\section{Children with FASD and typically developing controls showed differential DNA methylation patterns}

Following quality control and normalization, 431,544 sites of the 485,512 sites remained in the final dataset of 48 samples, which were corrected for batch effects using ComBat. While BECs are mostly a homogeneous population of cells, they contain small proportions of CD34and CD14-positive white blood cells, which can potentially skew DNA methylation analyses. As such, cell type deconvolution was performed to identify any blood contamination in the samples, identifying a trend towards significance in the proportions of different cells types between groups (CD34+, $p=0.115$; CD14+, $p=0.224$; BEC, $p=0.068$ ). To account for this factor in addition to other potential confounding variables within the dataset, we performed SVA to identify patterns of variation, identifying 6 surrogate variables when protecting the effects of group (FASD vs control). These were correlated with known sources of variation within the data, including cell type proportions and age (Additional file 2: Figure S1).

To identify DNA methylation patterns specific to the FASD group, we coupled differential DNA methylation analysis using a two-group design with the surrogate variables to correct for undesirable variation in the data. Given that we already accounted for ethnicity-related probes as much as possible in the NDN study, it was concluded that the effects of ethnic background would be lessened by using the final 658 differentially methylated CpGs [36]. As such, we performed linear modeling on the probes that were differentially methylated in the 
first study and remained in the dataset after preprocessing (648 CpGs of 658 from NDN). Of these, 161 CpGs displayed statistically significant differential methylation in the same direction as the initial cohort in the KBHN FASD group compared to the controls at an FDR $<0.05$ (Fig. 1a; Additional file 1: Table S2). To assess the probability of validating this many probes, random group subsampling was performed 10,000 times. As none showed more differentially methylated probes than the original replication cohort (maximum $=31$ differentially methylated probes), the probability of validating 161/648 probes was < 1e-4 (Additional file 2: Figure S2). Of the 161 validated probes, 82 were upmethylated, while 79 were down-methylated in FASD compared to control samples. Several genes contained multiple differentially methylated CpGs across both cohorts, including Hla-dpb1 (5), Fam59b (4), Capn10 (3), Des (3), Slc6a3 (3), Slc38a2 (3), Fam24a (2), H19 (2), and Tgfb1i1 (2) (Table 2). Moreover, 53 CpGs showed > $5 \%$ difference in methylation, an arbitrary cutoff often used to gauge potential biological significance [49]. Three genes contained 2 or more differentially methylated $(\mathrm{DM})$ probes that showed both an FDR $<0.05$ and $>5 \%$ difference in percent methylation, including Fam59b (4 probes), Hla-dpb1 (2 probes), and Slc6a3 (2 probes). In particular, the Fam59b CpGs were located
Table 2 Genes containing multiple differentially methylated CpGs in FASD

\begin{tabular}{lll}
\hline Gene & No. of CpGs & Direction of change \\
\hline Hla-dpb1 & 5 & Up \\
Fam59b & 4 & Down \\
Des & 3 & Down \\
Slc6a3 & 3 & Up \\
Slc38a2 & 3 & Down \\
Capn10 & 3 & Up \\
Fam24a & 2 & Up \\
H19 & 2 & Down \\
Tgfb1i1 & 2 & Down \\
\hline
\end{tabular}

within a CpG island and showed substantial differences in DNA methylation levels between FASD and control groups, with an average $13 \%$ methylation difference across the array probes in the CpG island (Fig. 2). Three additional sites located in intergenic regions showed $>10 \%$ percent DNA methylation difference between groups.

Overall, the percent methylation differences between groups of the 648 analyzed probes were highly correlated between the NDN and KBHN cohorts, as determined by linear modeling $(r=0.638, p<2.2 \mathrm{e}-16$; Fig. 1b). We also compared the ranking of probes by $p$ value from linear
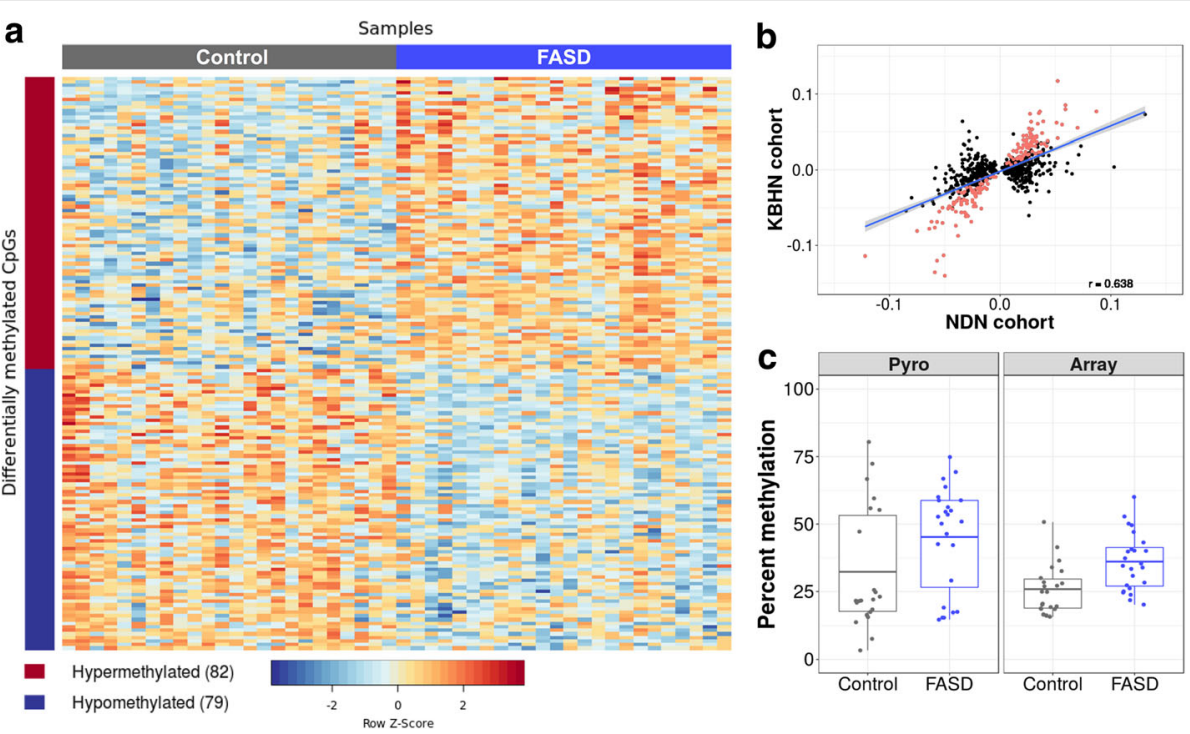

Fig. 1 Visualization and verification of the differentially methylated probes. a Heatmap of the 161 validated probes validated in the KBHN cohort at an FDR < 0.05 (79 hypermethylated in FASD; 82 hypomethylated in FASD). The percent methylation values (ranging from 0 to 100) were centered, scaled, and trimmed, resulting in a standardized DNA methylation level ranging from -2 to +2 (blue-red scale). $\mathbf{b}$ Scatter plot of the differences in percent methylation between FASD and controls for the 648 differentially probes identified in the NDN cohort. The mean differences between groups were highly correlated between both the NDN and KBHN cohorts $(r=0.638, p<2.2 \mathrm{e}-16)$. The red points show the probes that were statistically significant $(F D R<0.05)$ and showed the same direction of change across both studies $\mathbf{c}$ Verification by bisulfite pyrosequencing in FASD (blue) and control (gray) samples. The left panel shows the DNA methylation levels from the pyrosequencing assay, while the right panel shows the results from the 450K array. The CpG assayed was located in the CACNA1A gene body (cg24800175) and showed statistically significant differences between groups $(p=0.04)$ 


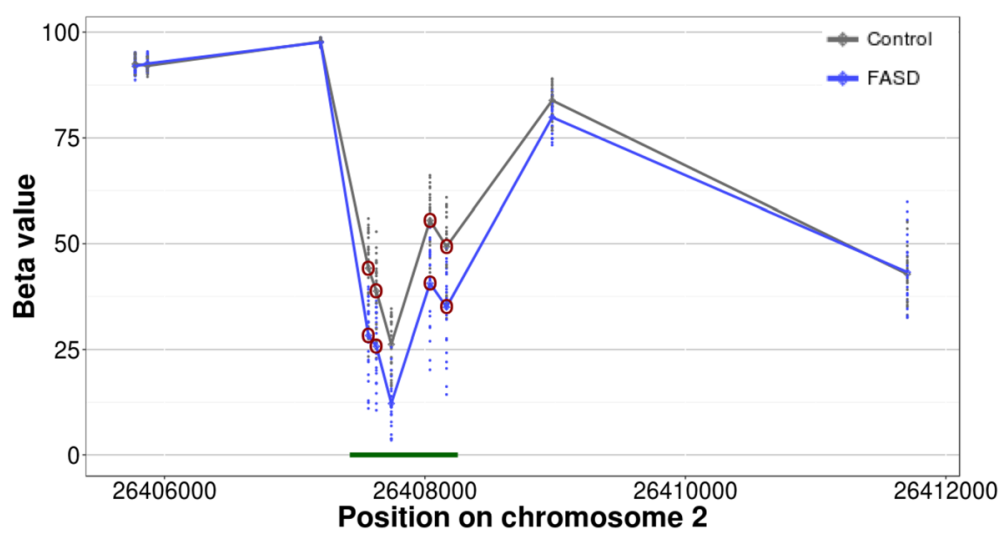

Fig. 2 Several differentially methylated CpGs were located in the Fam59b gene body. DNA methylation levels for FASD (blue) and controls (gray) are shown for $10 \mathrm{CpGs}$ within the gene, with the red circles representing the validated hits in KBHN (FDR $<0.05)$. These were located in a CpG island, illustrated by the green bar at the bottom, which showed an average 13\% difference in DNA methylation levels in individuals with FASD versus controls across all five CpGs covered by the 450K array

modeling between the NDN and KBHN cohorts; no significant similarities were identified $(p=0.91)$. Of note, 21 of the significant probes with $>5 \%$ methylation difference between groups from the NDN study were validated in the present analysis (39 of 41 were present in the filtered KBHN dataset). This proportion (54\%) was much higher than all validated probes (25\%), suggesting that these potentially represented more robust associations with FASD.

\section{Bisulfite pyrosequencing verified the differential DNA methylation of CACNA1A}

To verify that the differential DNA methylation results did not depend on the method used to measure them, we assessed DNA methylation levels of the cg24800175 probe in CACNA1A. We selected this probe as it was also verified in the initial NDN study, where it similarly showed a $>5 \%$ difference in DNA methylation between individuals with FASD and controls. Pyrosequencing results confirmed the DNA methylation levels observed on the $450 \mathrm{~K}$ array, showing similar DNA methylation levels and differences between groups for CpGs located in CACNA1A (Fig. 1c). The Pearson correlation between these two methods was 0.826 and the Bland-Altman plot showed little difference when comparing the $450 \mathrm{~K}$ array to pyrosequencing, suggesting good concordance between DNA methylation data from the two methods (Additional file 2: Figure S3). Linear regression analysis of pyrosequencing data between FASD cases and controls confirmed differential DNA methylation in this site, even without correcting for covariates $(p=0.04)$.

\section{DNA methylation patterns classified individuals with FASD versus controls}

To assess whether DNA methylation data could be used to predict FASD status, we created a predictive algorithm of FASD using machine learning approaches. First, we selected the normalized DNA methylation data (beta-values) of 179 samples from the NDN cohort (83 FASD; 96 control) in the 648 initial probes that were also found in the KBHN data. Our strategy was to build the predictor using an initial training cohort (NDN), followed by subsequent evaluation in the test cohort (KBHN). See Fig. 3 for an overview of steps used to build the FASD predictor.

Using a gradient boosting model in the caret package to optimize both sensitivity and specificity (area under the receiver operating characteristic (ROC) curve), we

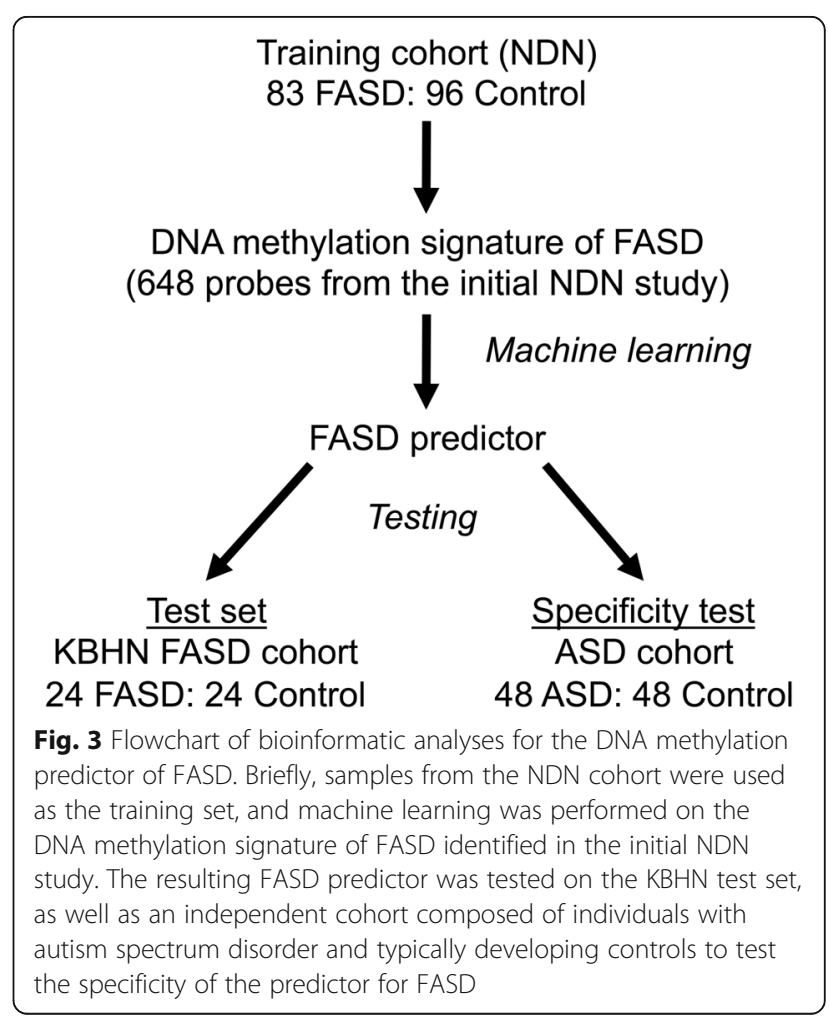


created a predictive model to assess the probability of FASD based on DNA methylation patterns [48]. This method provided weighted values for the different features (CpGs) of the model to determine their importance in classifying the samples. Of the 648 initial features, 183 had non-zero influence on the predictive model and could be used to predict FASD status (Additional file 1: Table S3). As the number of non-zero features was similar to the total number of samples, concerns of model over-fitting were reduced.

Through this approach, the predicted sensitivity and specificity for the training cohort were 0.879 and 0.944 , respectively, for an area under the curve of 0.977 (95\% confidence intervals, 0.972-0.982; Fig. 4a). The performance of the predictor on the training data indicated that DNA methylation could be used to distinguish FASD cases and controls, although these results will need to be carefully assessed in independent test sets or clinical settings.

To get a better understanding of the utility of this tool, we next assessed the predictive model using the normalized, batch-corrected DNA methylation data of the KBHN cohort as a test set. Of note, these data were not corrected for any covariates or surrogate variables other than batch correction. As expected for analysis of an independent test set, the model performed at a lower level in this cohort, displaying 0.917 sensitivity, 0.75 specificity, and 0.920 area under the ROC curve (Table 3; Fig. 4a). The balanced accuracy of the model in this cohort was 0.833 (95\% CI $0.698-0.925)$, and the ROC curve was not significantly different from the one obtained in the training cohort ( $p$ $=0.192$ ). Overall, 2 controls were misclassified as FASD and 6 children with FASD were misclassified as controls, giving a negative predictive value (NPV) of $78.6 \%$ and a positive predictive value (PPV) of $90 \%$. Given the discrepancies in ethnic backgrounds between FASD and control groups, the misclassified samples were assessed for differences in self-reported ethnicity, caregiver status, age, and buccal cell-type proportions in the classification. We did not identify any skew of these data in the misclassified controls, which were both Caucasian males aged 15 and 16, respectively. Although every misclassified individual with FASD had a previous diagnosis of ARND, a category that was present in high proportion within this cohort, no other patterns emerged between the correctly and incorrectly classified individuals with FASD ( 3 females/ 3 males; 3 First Nations/1 Métis/2 Caucasian; aged 6-18). Taken together, these findings suggested that differences in these demographic variables between the groups did not drive their classification.

The DNA methylation predictors were not biased by ASD in an independent cohort

BEC samples from an independent published autism spectrum disorder (ASD) cohort were used to assess the specificity of the model in the FASD cohorts. To this end, we used a publically available dataset of $450 \mathrm{~K}$ array data from the BECs of 48 individuals with ASD and 48 typically developing controls from the gene expression omnibus (GSE50759) [47]. Using processed GEO data from this cohort, the predictor correctly identified the vast majority of individuals in the cohort as non-FASD. The model only misclassified 1 individual as FASD, for a specificity of 0.990 (95\% CI 0.943-0.9997), higher than the predicted specificity in the training set. This sample, a 3-year-old female with ASD (51\% African ancestry, $41 \%$ European ancestry) did not have any particular distinguishing features compared to the correctly classified samples, suggesting that the predictive model was not biased for ASD, sex, age, or African ancestry in this independent cohort.

\section{Discussion}

Epigenetic marks are emerging as potential biomarkers and mediators of environmental exposures, and a growing body of literature suggests that epigenetic factors may be involved in the etiology of FASD. In particular, our recent study using a large cohort of children with FASD to date identified a signature of 658 differentially methylated CpGs in the BEC of individuals with FASD compared to typically developing controls [36]. Here, we present a validation of genome-wide DNA methylation data in a small cohort of individuals with FASD, where we successfully replicated 161 of the 658 differentially methylated CpGs identified in the initial NDN cohort. Furthermore, we demonstrated that DNA methylation data could be utilized to generate a predictive algorithm to classify individuals as FASD or control with high accuracy. These results indicated that DNA methylation in BECs could potentially be used towards developing a screening tool for children at risk for FASD.

Our present findings represent the initial validation of genome-wide DNA methylation differences in individuals with FASD. Of the 161 validated $\mathrm{CpGs}$ at an FDR < $0.05,53$ had $>5 \%$ difference in DNA methylation levels between groups. This $5 \%$ threshold is often used for assessing potential biological relevance in epigenetic studies of psychiatric and neurodevelopmental disorders, and therefore, we confined our interpretation of possible functional implications to CpGs with this effect size [47, 49-51]. Importantly, the biological significance of a $5 \%$ difference in DNA methylation is poorly understood, and its functional relevance may be limited in relation to gene expression or cellular physiology. Nevertheless, 21 CpGs showed a $>5 \%$ difference between FASD cases and controls at an FDR $<0.05$ in both the KBHN and NDN cohorts, suggesting that these may represent the strongest associations with FASD. Although the DNA methylation differences between FASD and controls 


\section{a Receiver operating characteristic curve}

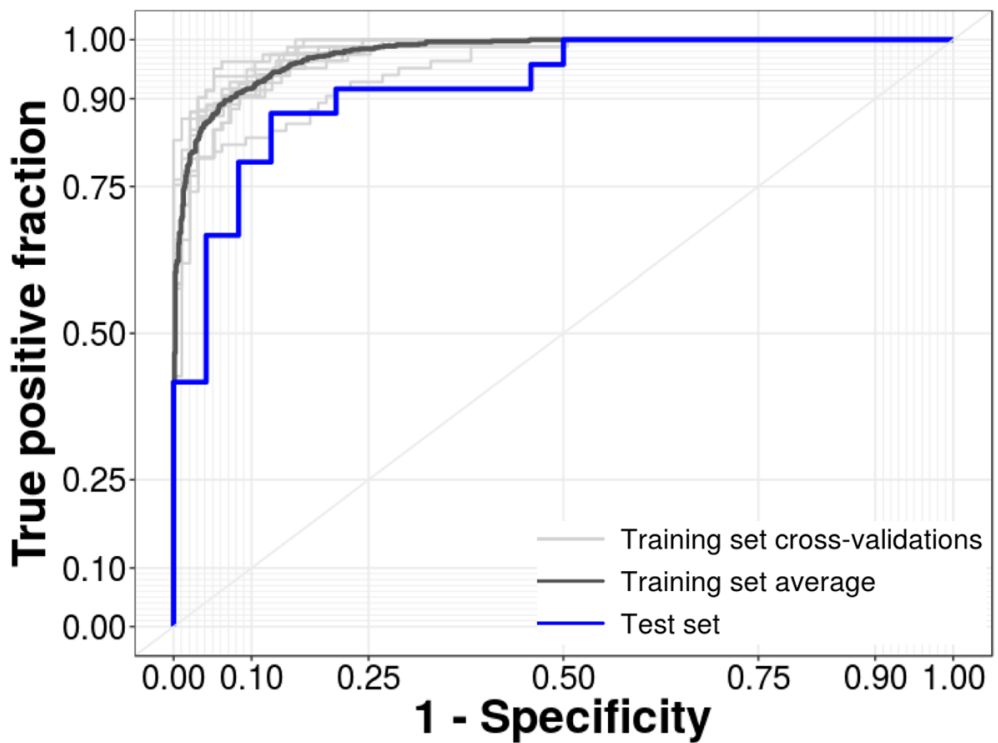

b $\quad$ Test set confusion matrix

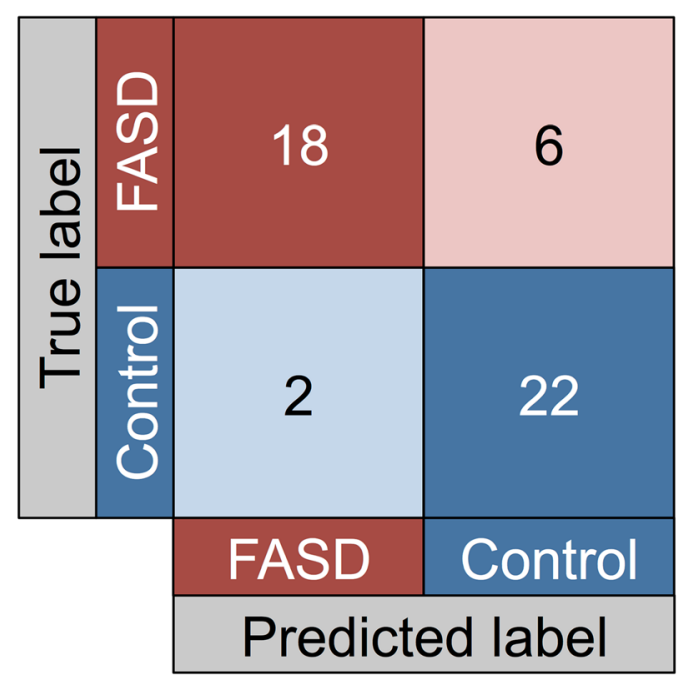

Fig. 4 Visualization of the training and test set performance for the DNA methylation predictor of FASD. a The DNA methylation predictor created using the 648 probes identified in NDN showed high accuracy in the training cohort (dark gray; area under the curve $=0.977$ ) and slightly poorer accuracy in the KBHN test set (blue; area under the curve $=0.920$ ). These curves were not significantly different $(p=0.192)$. $\mathbf{b}$ The confusion matrix displays number of samples classified correctly or incorrectly. Of note, six individuals with FASD in the test set were classified as controls, while only two control samples were misclassified as FASD

were highly correlated between the NDN and KBHN cohorts $(p<2.2 \mathrm{e}-16)$, the majority of CpGs showing the same direction of change did not achieve statistical significance $(301 / 648)$, potentially due to the replication cohort's small size or the absence of individuals with only PAE in this cohort. In addition, we verified the results from the $450 \mathrm{~K}$ array by bisulfite pyrosequencing, confirming the differential DNA methylation results for a CPG located in CACNA1A and supporting that our findings were not an artifact of array technology. As discussed in a recent comprehensive review, we note that the functional relevance of these differences is highly dependent on multiple factors, including subcellular differences, transcription factor binding regulation, density and cooperativity of DNA methylation, or other cis-regulatory elements [52].

Several genes previously associated with PAE or FASD contained multiple differentially methylated CpGs with $>5 \%$ difference in DNA methylation between groups, including Fam59b, Hla-dpb1, and Slc6a3. The Hla-dpb1 
Table 3 Summarized results from the classification algorithm

\begin{tabular}{ll}
\hline Training set (NDN) & \\
AUC & 0.977 \\
Accuracy & 0.914 \\
Sensitivity & 0.879 \\
Specificity & 0.944 \\
Test set (KBHN) & \\
AUC & 0.920 \\
Accuracy & 0.833 \\
Sensitivity & 0.75 \\
Specificity & 0.917 \\
False positives & 2 \\
False negatives & 6 \\
PPV & 0.900 \\
NPV & 0.786 \\
Negative control (ASD) & \\
Accuracy & 0.990 \\
Sensitivity & $\mathrm{NA}$ \\
Specificity & 0.990 \\
False positives & 1 \\
\hline
\end{tabular}

locus, a member of the major histocompatibility complex proteins, contained several differentially methylated CpGs, which overlapped with a differentially methylated region identified in the NDN study. Given its important function in immune regulation and potential role in rheumatoid arthritis, these differences could potentially reflect some of the immune deficits associated with FASD [53]. Furthermore, the Fam59b gene contained several CpGs with substantial (>10\%) differences in DNA methylation levels between individuals with FASD and controls, potentially representing a particularly sensitive locus with regard to FASD. Of note, only one validated $\mathrm{CpG}$ was located in one of the protocadherin gene clusters (Pcdhb18), which were considerably enriched in previous genome-wide studies of DNA methylation in individuals with FASD $[35,36]$. Given that these different studies only showed one overlapping probe, this could indicate higher variability within these gene clusters that may be associated with other variables not present in the current dataset, such as differences in age, body mass index, ethnicity, and socio-economic status.

Of particular interest, we replicated the differential DNA methylation patterns of the two genes involved in dopamine signaling from the NDN cohort, the dopamine transporter Slc6a3 and the dopamine receptor D4 (Drd4). Given the important role of the dopaminergic system in brain development and its interactions with neuroendocrine and immune systems, these differences could potentially reflect broader alterations to signaling pathways in the organism. Of note, the BEC of children exposed to alcohol during prenatal life and breastfeeding also display altered DNA methylation patterns in the promoter region of Drd4 [34]. Furthermore, several disorders previously associated with allelic variation and DNA methylation in this gene show either overlaps or co-morbidities with FASD, including attention deficit hyperactivity disorder, bipolar disorder, anxiety disorder, schizophrenia, and substance abuse [54-64]. Although it is tempting to interpret these findings in the context of PAE-induced deficits, DNA methylation differences in BEC likely do not fully reflect alterations in the central nervous system. Nevertheless, it has been suggested that BECs may act as a suitable surrogate tissue in human studies of DNA methylation, as they are also derived from the ectoderm [65]. While we did not measure these genes in additional tissues, evidence from animal models suggests that PAE can cause lasting alterations to the epigenome of central nervous system tissues, and as such, these results could potentially represent broader associations with epigenomic patterns in the brain [27].

Although these findings represent the initial validation of genome-wide DNA methylation data in children with FASD, a few particularities of the KBHN cohort limit the interpretability and generalizability of these results. Similar to the original cohort, the KBHN replication cohort was confounded by ethnicity, as the vast majority of FASD cases were from First Nations communities, while controls were mainly Caucasian. Given that genetic background influences DNA methylation patterns, differences between groups may have been, at least partly, due to ethnicity. Unfortunately, the KBHN cohort was too small to separate the groups into more ethnically homogeneous subsets, a method we had previously used to account for ethnicity-related differences in DNA methylation [37]. As such, we performed linear modeling on the sites that had been previously identified in the NDN study, which were partially filtered for ethnicityrelated differences during the analysis of the first cohort. However, some of the top differentially methylated genes could potentially be influenced by ethnicity differences between groups in spite of our best efforts. For instance, three known polymorphisms are located within the Fam59b locus (dbSNP minor allele frequencies: rs774397935, 1.04\%; rs4665833, 5.1\%; rs181971256, $21.4 \%)$. Although, as of now, none of these are known methylation quantitative trait loci (mQTL), the Fam59b gene body contains several mQTLs in the developing human brain, and genetic variation outside the region could potentially influence DNA methylation levels [66]. In addition, nearby genetic variation can also influence DNA methylation patterns in the promoter of $\operatorname{Drd4}$, which may be reflected in this cohort through the skew in ethnicity between groups [59]. Although the 
frequencies of these alleles in First Nation populations have not been assessed, genetic differences between groups could potentially influence DNA methylation levels within this differentially methylated region.

In addition to self-declared ethnicity, significant differences in the primary caregiver were present between groups, as all controls lived with their biological families, while the majority of children with FASD were generally in foster care or adoptive families. While the effects of this disparity on the epigenome are unclear, they could influence DNA methylation patterns through a number of factors, including nutrition, early-life adversity, and socio-economic status [67]. Individuals with FASD also tend to have life experiences different from those of typically developing children, which include early life adversity (e.g., maltreatment or neglect), separation from the biological family/placement in foster care (as occurred in our cohorts), poverty, and familial stress [13, 68]. Importantly, both pre- and postnatal experiences are known to play a role in early programming and thus may also influence DNA methylation patterns. As such, it may be difficult to separate the impacts of PAE and environmental adversity, and studies evaluating FASD may in many instances assess a combination of different factors and exposures, which is often the reality in this population. Nevertheless, our findings demonstrated clear and replicable associations between FASD and DNA methylation patterns across two independent cohorts. We believe that our use of SVA to partially account for unknown factors that could influence DNA methylation reduced some of the potential confounds associated with the cohort design. Future studies with larger groups that are balanced for ethnicity, age, and additional variables, including a focus on environmental stress/adversity, will be necessary to tease out these differences and further validate our findings.

Finally, we show here that DNA methylation patterns can be utilized as predictive variables for FASD in clinical populations. These findings complement and extend previous studies that investigated different molecular and physiological markers to help screen children for potential prenatal alcohol exposure, including alcohol metabolites in mothers and children, circulating miRNA in mothers, and cardiac orienting response in children [69-72]. In particular, eye tracking measures have been used in a small cohort of children to distinguish children with FASD, ADHD, or typically developing controls with relatively good accuracy [73]. In contrast to these studies, we selected only individuals with confirmed FASD from the initial NDN training cohort to create a DNA methylation-based predictor that was specific to individuals with an FASD diagnosis. The classification model was tuned to screen children at a higher risk for FASD with high sensitivity and specificity in an attempt to balance the false-positive and false-negative rates. Importantly, our results suggest that DNA methylation predictors can achieve high accuracy in the classification of individuals with FASD versus controls across multiple cohorts. Moreover, the predictive algorithm appeared to be largely independent of typical confounding factors, such as age, sex, ethnicity, and cell type composition of the samples. The predictor was also unbiased towards individuals with ASD and although there was no report of FASD in this independent cohort, it is possible that our reported false-positive could be due to an undiagnosed FASD case [74]. Collectively, these results support the use of DNA methylation as a potential screening tool for FASD.

\section{Conclusions}

Given the broad spectrum of cognitive, behavioral, and biological deficits associated with PAE, FASD places an important strain on both societal resources and the affected individuals and families. As such, accurate screening methods are necessary to identify children at risk for FASD at an early age, when interventions are most effective. Our findings provide an initial steppingstone towards epigenetic biomarkers of FASD and could potentially be adapted for the development of related screening tools for neurodevelopmental disorders. Validation of these tools across different cohorts, with increased sample sizes, varying ages, ethnicities, and better documented environmental exposures will be essential to parse out the strongest associations and to develop reliable epigenetic screening tools for FASD.

\section{Additional files}

Additional file 1: Supplementary tables. (XLSX $69 \mathrm{~kb}$ )

Additional file 2: Supplementary figures. (DOCX 130 kb)

\section{Abbreviations}

450K array: Illumina HumanMethylation450 BeadChip array; ARND: Alcoholrelated neurodevelopmental disorder; ASD: Autism spectrum disorder; BEC: Buccal epithelial cell; CpG: Cytosine-guanine dinucleotide;

DNA: Deoxyribonucleic acid; FAS: Fetal alcohol syndrome; FASD: Fetal alcohol spectrum disorder; FDR: False discovery rate; GEO: Gene Expression Omnibus; KBHN: Kids Brain Health Network; NDN: NeuroDevNet;

PAE: Prenatal alcohol exposure; PCDH: Protocadherin; RNA: Ribonucleic acid; ROC: Receiver operator characteristic; SV: Surrogate variable; SVA: Surrogate variable analysis

\section{Acknowledgements}

MSK is the Canada Research Chair in Social Epigenetics, the Sunny Hill BC Leadership Chair in Child Development, and a Senior Fellow of the Canadian Institute For Advanced Research (CIFAR).

\section{Funding}

This research is supported by the Kids Brain Health Network (formerly NeuroDevNet), a Canadian Network of Center's for Excellence and a program of the federal government to advance science and technology. AAL is supported by a Developmental Neurosciences Research Training award from Brain Canada \& NeuroDevNet. JW is supported by grants from the US 
National Institutes of Health/National Institute on Alcohol Abuse and Alcoholism (R37 AA007789 and RO1 AA022460); and the Canadian Foundation for Fetal Alcohol Research. PP is supported by an NSERC Discovery Grant.

\section{Availability of data and materials}

The KBHN DNA methylation data are deposited into the GEO database under accession number GSE109042. The predictive algorithm can be found and used here: https://fasdpredictor.shinyapps.io/fasdpredictorapp/.

\section{Authors' contributions}

AAL all bioinformatic analyses and wrote the manuscript. AMM performed the pyrosequencing. JS and AEC collected the samples. AEC, JW, PP, JNR, and MSK helped with the study design, interpretation, and writing. All authors read and approved the final manuscript.

\section{Ethics approval and consent to participate}

Ethics for this study were reviewed and approved by the "Children's and Women's Research Ethics Board - Clinical" (H10-01149). All experimental procedures were reviewed and approved by the University of Manitoba and the University of British Columbia.

\section{Consent for publication}

Written informed consent was obtained from a parent or legal guardian and assent was obtained from each child before study participation.

\section{Competing interests}

The authors declare that they have no competing interests.

\section{Publisher's Note}

Springer Nature remains neutral with regard to jurisdictional claims in published maps and institutional affiliations.

\section{Author details}

${ }^{1}$ Department of Medical Genetics, Centre for Molecular Medicine and Therapeutics, British Columbia Children's Hospital Research Institute, University of British Columbia, Vancouver, British Columbia, Canada. ${ }^{2}$ Department of Cellular and Physiological Sciences, Life Sciences Institute, University of British Columbia, Vancouver, British Columbia, Canada. ${ }^{3}$ Department of Pediatrics and Child Health, Faculty of Medicine, University of Manitoba, Winnipeg, Manitoba, Canada. ${ }^{4}$ Department of Biochemistry and Medical Genetics, Faculty of Medicine, University of Manitoba, Winnipeg, Manitoba, Canada. ${ }^{5}$ Department of Biomedical and Molecular Sciences, Centre for Neuroscience Studies, Queen's University, Kingston, Ontario, Canada. ${ }^{6}$ Michael Smith Laboratories, University of British Columbia, Vancouver, British Columnbia, Canada. ${ }^{7}$ Human Early Learning Partnership, University of British Columbia, Vancouver, British Columbia, Canada. ${ }^{8}$ Department of Psychiatry, University of British Columbia, Vancouver, British Columbia, Canada.

Received: 22 August 2017 Accepted: 4 January 2018

Published online: 12 January 2018

\section{References}

1. May PA, Gossage JP, Kalberg WO, Robinson LK, Buckley D, Manning M, et al. Prevalence and epidemiologic characteristics of FASD from various research methods with an emphasis on recent in-school studies. Dev Disabil Res Rev. 2009;15:176-92.

2. May PA, Baete A, Russo J, Elliott AJ, Blankenship J, Kalberg WO, et al. Prevalence and characteristics of fetal alcohol spectrum disorders. Pediatrics. 2014;134:855-66. https://doi.org/10.1542/peds.2013-3319.

3. May PA, Keaster C, Bozeman R, Goodover J, Blankenship J, Kalberg WO, et al. Prevalence and characteristics of fetal alcohol syndrome and partial fetal alcohol syndrome in a Rocky Mountain Region City. Drug Alcohol Depend. 2015;155:118-27. https://doi.org/10.1016/j.drugalcdep.2015.08.006

4. Zhang $X$, Sliwowska JH, Weinberg J. Prenatal alcohol exposure and fetal programming: effects on neuroendocrine and immune function. Exp Biol Med. 2005;230:376-88.

5. Pei J, Denys K, Hughes J, Rasmussen C. Mental health issues in fetal alcohol spectrum disorder. J Ment Health. 2011;20:473-83.
6. Mattson SN, Crocker N, Nguyen TT. Fetal alcohol spectrum disorders: neuropsychological and behavioral features. Neuropsychol Rev. 2011:21:81-101.

7. Jones $\mathrm{KL}$, Smith DW. Recognition of the fetal alcohol syndrome in early infancy. Lancet. 1973;302:999-1001. https://doi.org/10.1016/S01406736(73)91092-1.

8. Astley SJ, Clarren SK. Diagnosing the full spectrum of fetal alcoholexposed individuals: introducing the 4-digit diagnostic code. Alcohol Alcohol. 2000;35:400-10.

9. Jacobson SW, Jacobson $J$, Stanton ME, Meintjes EM, Molteno CD. Biobehavioral markers of adverse effect in fetal alcohol spectrum disorders. Neuropsychol Rev. 2011;21:148-66.

10. Pollard I. Neuropharmacology of drugs and alcohol in mother and fetus. Semin Fetal Neonatal Med. 2007:12:106-13.

11. Senturias $Y$, Baldonado M. Fetal spectrum disorders: an overview of ethical and legal issues for healthcare providers. Curr Probl Pediatr Adolesc Health Care. 2014;44:102-4. doi:https://doi.org/10.1016/j.cppeds.2013.12.010

12. Paley $\mathrm{B}, \mathrm{O}^{\prime}$ Connor MJ. Behavioral interventions for children and adolescents with fetal alcohol spectrum disorders. Alcohol Res Heal. 2011;34:64-75. http://www.ncbi.n/m.nih.gov/pmc/articles/PMC3860556/

13. Streissguth AP, Bookstein F, Barr H, Sampson P, O'Malley K, Young J. Risk factors for adverse life outcomes in fetal alcohol syndrome and fetal alcoho effects. J Dev Behav Pediatr. 2004;25:228-38. https://doi.org/10.1097/ 00004703-200408000-00002.

14. Fox SE, Levitt P, Nelson CA III. How the timing and quality of early experiences influence the development of brain architecture. Child Dev. 2010;81:28-40. https://doi.org/10.1111/j.1467-8624.2009.01380.x.

15. Russell M, Martier SS, Sokol RJ, Mudar P, Jacobson S, Jacobson J. Detecting risk drinking during pregnancy: a comparison of four screening questionnaires. Am J Public Health. 1996;86:1435-9. http://www.ncbi.nlm. nih.gov/pmc/articles/PMC1380656/

16. Jones TB, Bailey BA, Sokol RJ. Alcohol use in pregnancy: insights in screening and intervention for the clinician. Clin Obstet Gynecol. 2013;56: 114-23. https://doi.org/10.1097/GRF.0b013e31827957c0.

17. Burns E, Gray R, Smith LA. Brief screening questionnaires to identify problem drinking during pregnancy: a systematic review. Addiction. 2010;105:601-14. https://doi.org/10.1111/j.1360-0443.2009.02842.x.

18. Concheiro-Guisan A, Concheiro M. Bioanalysis during pregnancy: recent advances and novel sampling strategies. Bioanalysis. 2014;6:3133-53. https:// doi.org/10.4155/bio.14.278

19. Cabarcos P, Álvarez I, Tabernero MJ, Bermejo AM. Determination of direct alcohol markers: a review. Anal Bioanal Chem. 2015;407:4907-25. https://doi. org/10.1007/s00216-015-8701-7.

20. Bird A. Perceptions of epigenetics. Nature. 2007;447:396-8.

21. Meaney MJ. Epigenetics and the biological definition of gene $X$ environment interactions. Child Dev. 2010:81:41-79.

22. Henikoff S, Greally JM. Epigenetics, cellular memory and gene regulation. Curr Biol. 2016;26:R644-8. http://dx.doi.org/10.1016/j.cub.2016.06.011

23. Boyce WT, Kobor MS. Development and the epigenome: the "synapse" of gene-environment interplay. Dev Sci. 2015;18:1-23. https://doi.org/10.1111/ desc.12282.

24. Joubert BR, Håberg SE, Nilsen RM, Wang X, Vollset SE, Murphy SK, et al. 450K epigenome-wide scan identifies differential DNA methylation in newborns related to maternal smoking during pregnancy. Environ Health Perspect. 2012;120:1425-31. https://doi.org/10.1289/ehp.1205412.

25. Heijmans BT, Tobi EW, Stein AD, Putter H, Blauw GJ, Susser ES, et al. Persistent epigenetic differences associated with prenatal exposure to famine in humans. Proc Natl Acad Sci U S A. 2008;105:17046-9. https://doi. org/10.1073/pnas.0806560105.

26. Reese SE, Zhao S, Wu MC, Joubert BR, Parr CL, Håberg SE, et al. DNA Methylation score as a biomarker in newborns for sustained maternal smoking during pregnancy. Environ Health Perspect. 2017;125:760-6. https://doi.org/10.1289/EHP333.

27. Lussier AA, Weinberg J, Kobor MS. Epigenetics studies of fetal alcohol spectrum disorder: where are we now? Epigenomics. 2017:9:291-311. https://doi.org/10.2217/epi-2016-0163.

28. Chater-Diehl EJ, Laufer BI, Castellani CA, Alberry BL, Singh SM. Alteration of gene expression, DNA methylation, and histone methylation in free radica scavenging networks in adult mouse hippocampus following fetal alcohol exposure. PLoS One. 2016;11:e0154836.

29. Laufer BI, Mantha K, Kleiber ML, Diehl EJ, Addison SMF, Singh SM. Longlasting alterations to DNA methylation and ncRNAs could underlie the 
effects of fetal alcohol exposure in mice. Dis Model Mech. 2013;6:977-92. https://doi.org/10.1242/dmm.010975.

30. Liu Y, Balaraman Y, Wang G, Nephew KP, Zhou FC. Alcohol exposure alters DNA methylation profiles in mouse embryos at early neurulation. Epigenetics. 2009;4:500-11.

31. Hicks SD, Middleton FA, Miller MW. Ethanol-induced methylation of cell cycle genes in neural stem cells. J Neurochem. 2010;114:1767-80.

32. Zhou FC, Chen Y, Love A. Cellular DNA methylation program during neurulation and its alteration by alcohol exposure. Birth Defects Res Part A Clin Mol Teratol. 2011;91:703-15.

33. Otero NKH, Thomas JD, Saski CA, Xia X, Kelly SJ. Choline supplementation and DNA methylation in the hippocampus and prefrontal cortex of rats exposed to alcohol during development. Alcohol Clin Exp Res. 2012;36:1701-9.

34. Fransquet PD, Hutchinson D, Olsson CA, Wilson J, Allsop S, Najman J, et al. Perinatal maternal alcohol consumption and methylation of the dopamine receptor DRD4 in the offspring: the triple B study. Environ Epigenetics. 2016; 2:dvw023. http://dx.doi.org/10.1093/eep/dvw023

35. Laufer BI, Kapalanga J, Castellani CA, Diehl EJ, Yan L, Singh SM. Associative DNA methylation changes in children with prenatal alcohol exposure. Epigenomics. 2015;7 August:1-16. https://doi.org/10.2217/epi.15.60.

36. Portales-Casamar E, Lussier AA, Jones MJ, Maclsaac JL, Edgar RD, Mah SM, et al. DNA methylation signature of human fetal alcohol spectrum disorder. Epigenetics Chromatin. 2016:9:81-101.

37. Portales-Casamar E, Lussier AA, Jones MJ, Maclsaac JL, Edgar RD, Mah SM, et al. DNA methylation signature of human fetal alcohol spectrum disorder. Epigenetics Chromatin. 2016;9:25. https://doi.org/10.1186/ s13072-016-0074-4

38. Reynolds JN, Weinberg J, Clarren S, Beaulieu C, Rasmussen C, Kobor M, et al. Fetal alcohol spectrum disorders: gene-environment interactions, predictive biomarkers, and the relationship between structural alterations in the brain and functional outcomes. Semin Pediatr Neurol. 2011;18:49-55.

39. Chudley AE, Conry J, Cook JL, Loock C, Rosales T, LeBlanc N. Fetal alcohol spectrum disorder: Canadian guidelines for diagnosis. Can Med Assoc J. 2005;172(5 Suppl):S1-21.

40. Price ME, Cotton AM, Lam LL, Farré P, Emberly E, Brown CJ, et al. Additional annotation enhances potential for biologically-relevant analysis of the Illumina Infinium HumanMethylation450 BeadChip array. Epigenetics Chromatin. 2013;6:4. https://doi.org/10.1186/1756-8935-6-4.

41. Teschendorff AE, Marabita F, Lechner M, Bartlett T, Tegner J, Gomez-Cabrero $D$, et al. A Beta-mixture quantile normalization method for correcting probe design bias in Illumina Infinium 450k DNA methylation data. Bioinformatics. 2012;29:189-96.

42. Leek JT, Johnson WE, Parker HS, Jaffe AE, Storey JD. The sva package for removing batch effects and other unwanted variation in high-throughput experiments. Bioinformatics. 2012;28:882-3.

43. Du P, Zhang X, Huang C-C, Jafari N, Kibbe WA, Hou L, et al. Comparison of Beta-value and M-value methods for quantifying methylation levels by microarray analysis. BMC Bioinformatics. 2010;11:587. https://doi.org/10. 1186/1471-2105-11-587.

44. Smith AK, Kilaru V, Klengel T, Mercer KB, Bradley B, Conneely KN, et al. DNA extracted from saliva for methylation studies of psychiatric traits: evidence tissue specificity and relatedness to brain. Am J Med Genet Part B Neuropsychiatr Genet. 2015;168:36-44.

45. Smyth GK. Linear models and empirical bayes methods for assessing differential expression in microarray experiments. Stat Appl Genet Mol Biol. 2004;3:Article3.

46. Benjamini Y, Hochberg Y. Controlling the false discovery rate: a practical and powerful approach to multiple testing. J R Stat Soc Ser B. 1995;57:289-300. https://doi.org/10.2307/2346101.

47. Berko ER, Suzuki M, Beren F, Lemetre C, Alaimo CM, Calder RB, et al. Mosaic epigenetic dysregulation of ectodermal cells in autism spectrum disorder. PLOS Genet. 2014;10:e1004402. https://doi.org/10.1371/journal.pgen. 1004402.

48. Kuhn M. Building predictive models in R using the caret package. J Stat Software. 2008;1(5) https://doi.org/10.18637/jss.v028.i05.

49. Breton CV, Marsit CJ, Faustman E, Nadeau K, Goodrich JM, Dolinoy DC, et al. Small-magnitude effect sizes in epigenetic end points are important in children's environmental health studies: the Children's environmental health and disease prevention research center's epigenetics working group. Environ Health Perspect. 2017;125:511-26. https://doi.org/10.1289/EHP595.
50. Ladd-Acosta C, Hansen KD, Briem E, Fallin MD, Kaufmann WE, Feinberg AP Common DNA methylation alterations in multiple brain regions in autism. Mol Psychiatry. 2014;19:862-71.

51. Rakyan VK, Down TA, Balding DJ, Beck S. Epigenome-wide association studies for common human diseases. Nat Rev Genet. 2011;12:529-41.

52. Lappalainen T, Greally JM. Associating cellular epigenetic models with human phenotypes. Nat Rev Genet. 2017;18:441-51. http://dx.doi.org/10. 1038/nrg.2017.32

53. Liu Y, Aryee MJ, Padyukov L, Fallin MD, Hesselberg E, Runarsson A, et al. Epigenome-wide association data implicate DNA methylation as an intermediary of genetic risk in rheumatoid arthritis. Nat Biotechnol. 2013;31: 142-7. https://doi.org/10.1038/nbt.2487.

54. Sánchez-Mora C, Ribasés M, Casas M, Bayés M, Bosch R, Fernàndez-Castillo $\mathrm{N}$, et al. Exploring DRD4 and its interaction with SLC6A3 as possible risk factors for adult ADHD: a meta-analysis in four European populations. Am J Med Genet Part B, Neuropsychiatr Genet. 2011;156B:600-12.

55. Dadds MR, Schollar-Root O, Lenroot R, Moul C, Hawes DJ. Epigenetic regulation of the DRD4 gene and dimensions of attention-deficit/ hyperactivity disorder in children. Eur Child Adolesc Psychiatry. 2016;25: 1081-9. https://doi.org/10.1007/s00787-016-0828-3.

56. Ji H, Wang $Y$, Jiang $D$, Liu G, Xu X, Dai D, et al. Elevated DRD4 promoter methylation increases the risk of Alzheimer's disease in males. Mol Med Rep. 2016;14:2732-8.

57. Cheng J, Wang Y, Zhou K, Wang L, Li J, Zhuang Q, et al. Male-specific association between dopamine receptor D4 gene methylation and schizophrenia. PLoS One. 2014;9:e89128. https://doi.org/10.1371/journal. pone.0089128

58. Kordi-Tamandani DM, Sahranavard R, Torkamanzehi A. Analysis of association between dopamine receptor genes' methylation and their expression profile with the risk of schizophrenia. Psychiatr Genet. 2013;23: 183-7. http://journals.Iww.com/psychgenetics/Abstract/2013/10000/ Analysis_of_association_between_dopamine_receptor.1.aspx.

59. Docherty SJ, Davis OSP, Haworth CMA, Plomin R, D'Souza U, Mill J. A genetic association study of DNA methylation levels in the DRD4 gene region finds associations with nearby SNPs. Behav Brain Funct. 2012:8:31. https://doi.org/10.1186/1744-9081-8-31.

60. Ptáček R, Kuželová H, Stefano GB. Dopamine D4 receptor gene DRD4 and its association with psychiatric disorders. Med Sci Monit. 2011;17:RA215-20. https://doi.org/10.12659/MSM.881925.

61. Bau CH, Almeida S, Costa FT, Garcia CE, Elias EP, Ponso AC, et al. DRD4 and DAT1 as modifying genes in alcoholism: interaction with novelty seeking on level of alcohol consumption. Mol Psychiatry. 2001;6:7-9.

62. Zhang H, Herman Al, Kranzler HR, Anton RF, Zhao H, Zheng W, et al. Arraybased profiling of DNA methylation changes associated with alcohol dependence. Alcohol Clin Exp Res. 2013;37(Suppl 1):E108-15.

63. Faraone SV, Bonvicini C, Scassellati C. Biomarkers in the diagnosis of ADHD--promising directions. Curr Psychiatry Rep. 2014;16:497. https://doi. org/10.1007/s11920-014-0497-1.

64. Chen D, Liu F, Shang Q, Song X, Miao X, Wang Z. Association between polymorphisms of DRD2 and DRD4 and opioid dependence: evidence from the current studies. Am J Med Genet Part B Neuropsychiatr Genet. 2011;156: 661-70. https://doi.org/10.1002/ajmg.b.31208.

65. Lowe R, Gemma C, Beyan H, Hawa MI, Bazeos A, Leslie RD, et al. Buccals are likely to be a more informative surrogate tissue than blood for epigenomewide association studies. Epigenetics. 2013;8:445-54.

66. Hannon E, Spiers H, Viana J, Pidsley R, Burrage J, Murphy TM, et al. Methylation QTLs in the developing brain and their enrichment in schizophrenia risk loci. Nat Neurosci. 2015;19:48-54. https://doi.org/10. 1038/nn.4182.

67. Esposito EA, Jones MJ, Doom JR, Maclsaac JL, Gunnar MR, Kobor MS. Differential DNA methylation in peripheral blood mononuclear cells in adolescents exposed to significant early but not later childhood adversity. Dev Psychopathol. 2016;28 4pt2:1385-99. https://doi.org/10.1017/ S0954579416000055

68. Coggins TE, Timler GR, Olswang LB. A state of double jeopardy: impact of prenatal alcohol exposure and adverse environments on the social communicative abilities of school-age children with fetal alcohol spectrum disorder. Lang Speech Hear Serv Sch. 2007;38:117-27. http://dx.doi.org/10. 1044/0161-1461(2007/012)

69. Balaraman S, Schafer JJ, Tseng AM, Wertelecki W, Yevtushok L, ZymakZakutnya $\mathrm{N}$, et al. Plasma miRNA profiles in pregnant women predict infant 
outcomes following prenatal alcohol exposure. PLoS One. 2016;11: e0165081. https://doi.org/10.1371/journal.pone.0165081.

70. Mesa DA, Kable JA, Coles CD, Jones KL, Yevtushok L, Kulikovsky Y, et al. The use of cardiac orienting responses as an early and scalable biomarker of alcohol-related neurodevelopmental impairment. Alcohol Clin Exp Res. 2017:41:128-38. https://doi.org/10.1111/acer.13261.

71. Goh PK, Doyle LR, Glass L, Jones KL, Riley EP, Coles CD, et al. A decision tree to identify children affected by prenatal alcohol exposure. J Pediatr. 2016; 177:121-127.e1. https://doi.org/10.1016/j.jpeds.2016.06.047

72. McQuire C, Paranjothy S, Hurt L, Mann M, Farewell D, Kemp A. Objective measures of prenatal alcohol exposure: a systematic review. Pediatrics. 2016; 138 https://doi.org/10.1542/peds.2016-0517.

73. Tseng P-H, Cameron IGM, Pari G, Reynolds JN, Munoz DP, Itti L. Highthroughput classification of clinical populations from natural viewing eye movements. J Neurol. 2013;260:275-84. https://doi.org/10.1007/ s00415-012-6631-2.

74. Kelleher E, Corvin A. Overlapping etiology of neurodevelopmental disorders. In: The genetics of Neurodevelopmental disorders: Wiley; 2015. p. 29-48. https://doi.org/10.1002/9781118524947.ch2.

\section{Submit your next manuscript to BioMed Central} and we will help you at every step:

- We accept pre-submission inquiries

- Our selector tool helps you to find the most relevant journal

- We provide round the clock customer support

- Convenient online submission

- Thorough peer review

- Inclusion in PubMed and all major indexing services

- Maximum visibility for your research

Submit your manuscript at www.biomedcentral.com/submit 